Gut and Liver, Vol. 12, No. 1, January 2018, pp. 79-85

\title{
Non-hypervascular Hypointense Nodules on Hepatocyte Phase Gadoxetic Acid-Enhanced MR Images: Transformation of MR Hepatobiliary Hypointense Nodules into Hypervascular Hepatocellular Carcinomas
}

\author{
Yun Ku Cho ${ }^{1}$, Ju Won $\mathrm{Kim}^{1}$, Mi Young $\mathrm{Kim}^{1}$, and Hyeon Je $\mathrm{Cho}^{2}$ \\ ${ }^{1}$ Department of Radiology, VHS Medical Center, Seoul, and ${ }^{2}$ Department of Radiology, International St. Mary's Hospital, Catholic Kwandong. \\ University, Incheon, Korea
}

Background/Aims: The annual risk of transformation of non-hypervascular magnetic resonance (MR) hepatobiliary phase imaging (HBPI) hypointense nodules into hypervascular hepatocellular carcinomas (HCCs) was evaluated. Methods: Cirrhotic patients with non-hypervascular HBPI hypointense nodules were retrospectively analyzed if they were diagnosed as HCC and MR followed up longer than 1 year during the period from January 2010 to October 2016 with regular intervals of 3 to 6 months. Risk factors for transformation into hypervascular HCCs were analyzed using the Cox proportional hazard model. Results: Among the 103 non-hypervascular HBPI hypointense nodules meeting the inclusion criteria, transformation into hypervascular HCCs occurred in 44 tumors (42.7\%). The median follow-up period was 24 months. Multivariate analysis revealed that hyperintensity on T2-weighted images (T2WI) and diffusionweighted images (DWI) were the two independent predictors of transformation into hypervascular HCCs $(p=0.036$ and $p=0.041$, respectively). Most tumors with hyperintensity on T2WI or DWI on the initial or follow-up MR were transformed into hypervascular HCCs within the first year. Among the 22 nodules (21.3\%) showing a new change in dynamic phases during follow-up, 14 nodules (13.6\%) showed malignant transformations. Conclusions: The transformation rates of HBPI hypointense nodules into hypervascular HCCs could be predicted according to the initial or serial MRI findings. (Gut Liver 2018;12:79-85)

Key Words: Liver neoplasms; Liver magnetic resonance; Carcinoma, hepatocellular; Malignant transformation

\section{INTRODUCTION}

Hepatocellular carcinoma (HCC) is the sixth most common cancer worldwide, and commonly occurs in patients at high risk for this disease, especially cirrhosis. As is well known, HCCs usually develop via the multistep process of hepatocarcinogenesis. ${ }^{1-3}$ In particular, high grade dysplastic nodules and pathologically early stage HCCs are known to be precursors of progressed HCCs, and can be regarded as borderline malignant nodules. ${ }^{2,4}$ In contrast to progressed HCCs, vascular invasion or intrahepatic metastasis is not likely to occur in these borderline malignant hepatic nodules. ${ }^{5,6}$ Therefore, detection of these hepatic nodules may play a role in reducing the possibility of tumor spread in cirrhotic patients. ${ }^{6,7}$

A recent study showed that hepatobiliary phase images (HBPI) of gadoxetic acid-enhanced magnetic resonance imaging (MRI) was superior to multidetector computed tomography or dynamic gadoxetic acid enhanced MRI in the detection of borderline malignant hepatic nodules. ${ }^{8}$ These nodules frequently appear as non-hypervascular hypointense nodules on HBPI because of the decrease or lack of uptake of gadoxetic acid, which is liverspecific MR contrast agent. ${ }^{9,10}$ Many of these precursor nodules are transformed into hypervascular HCCs during follow-up, ${ }^{8,11-13}$ and hyperintensity on T2 weighted images (T2WI) or diffusion weighted images (DWI) is known as a potent risk factor for the subsequent transformation., ${ }^{8,11,12}$

However, previous studies did not tell whether each nonhypervascular HBPI hypointense nodule will be eventually transformed into hypervascular HCCs, how fast the nodules will be transformed into hypervascular HCC, or whether additional survival benefits can be expected from the early treatment of non-hypervascular HBPI hypointense nodules. In this study, regular MR follow-up were made for these non-hypervascular

\footnotetext{
Correspondence to: Yun Ku Cho

Department of Radiology, VHS Medical Center, 53 Jinhwangdo-ro 61-gil, Gangdong-gu, Seoul 05368, Korea

Tel: +82-2-2225-1442, Fax: +82-2-2225-1488, E-mail: yunkucho2004@yahoo.co.kr

Received on January 24, 2017. Revised on March 10, 2017. Accepted on March 10, 2017. Published online August 14, 2017

pISSN 1976-2283 eISSN 2005-1212 https://doi.org/10.5009/gnl17046

@ This is an Open Access article distributed under the terms of the Creative Commons Attribution Non-Commercial License (http://creativecommons.org/licenses/by-nc/4.0) which permits unrestricted non-commercial use, distribution, and reproduction in any medium, provided the original work is properly cited.
} 
HBPI hypointense hepatic nodules to evaluate the risk of transformation into hypervascular HCC nodules.

\section{MATERIALS AND METHODS}

\section{Patients}

The requirement for written informed consent was waived, and Institutional Review Board in VHS Medical Center approved this study. The inclusion criteria for this study were as follows: (1) cirrhotic patients who were diagnosed as HCC with Barcelona Clinic Liver Cancer (BCLC) stage $A ;{ }^{14}$ (2) those who underwent radiofrequency ablation (RFA) or surgical resection as an initial treatment for the HCC nodules; (3) patients with one or multiple non-hypervascular HBPI hypointense nodules up to five in number; (4) those who underwent repetitive gadoxetic acidenhanced liver MRI with regular intervals of 6 months or less; (5) patients were included in the analysis if initial MR work-up was done during the period from January 2010 to July 2015; and (6) the overall MR follow-up period was longer than 1 year. Those patients with six or more hypovascular HBPI hypointense nodules were excluded from the analysis to minimize the effect of clustering on statistical tests.

When patients had a single nodular HCC or multiple nodules up to three, each less than or equal to $3 \mathrm{~cm}$, hepatic resection was considered as the choice of treatment. However, RFA was recommended as an alternative treatment modality instead of hepatic resection when clinically significant portal hypertension is suspected based on the presence of varices or splenomegaly associated with thrombocytopenia. ${ }^{14}$ Sometimes, RFA was performed against the recommendations for surgery by clinicians, because of the patient's preference. After May 2012, RFA was considered as the primary treatment modality in patients with a single nodular HCC less than $2 \mathrm{~cm}$ in diameter according to the current American Association for the Study of Liver Disease (AASLD) practice guidelines. ${ }^{14}$

\section{Diagnosis of HCC}

The diagnosis of HCC was made using the noninvasive criteria defined by the AASLD recommendations, which were arterial hyper-enhancement and washout on portal or delayed phase images of liver MRI and/or dynamic computed tomography. ${ }^{15}$ Pathologic confirmation of HCC diagnoses was made through liver biopsies in patients who did not meet the noninvasive diagnostic criteria.

\section{Acquisition of gadoxetic acid-enhanced liver MRI}

Gadoxetic acid-enhanced liver MRI was performed by using a 1.5T (Signa HDxt; GE Medical Systems, Milwaukee, WI, USA) or a 3.0T (Skyra; Siemens Medical Solutions, Erlangen, Germany) superconducting system with an 8-channel or a 32-channel phased-array coil, respectively. Our detailed gadoxetic acidenhanced liver MRI protocol is described in Table 1. All patients received a rapid bolus of $0.1 \mathrm{~mL} / \mathrm{kg}(0.025 \mathrm{mmol} / \mathrm{kg})$ body weight of gadoxetic acid at a rate of $1.0 \mathrm{~mL} / \mathrm{s}$, immediately followed by a $25 \mathrm{~mL}$ saline flushing through an antecubital venous catheter using a power injector.

The arterial phase (AP) was scanned 7 seconds after the contrast media had arrived at the distal thoracic aorta, which was monitored by using real-time MR fluoroscopy. Portal venous phase (PVP), early venous phase (EVP), late venous phase, and hepatobiliary phase (HBP) were subsequently scanned 50 seconds, 80 seconds, 2 minutes, and 20 minutes after starting the administration of contrast media, respectively. Subtraction images were obtained by subtracting precontrast images from AP images to further evaluate the enhancement pattern of nodules on AP images. ${ }^{16,17}$ The slice thickness of the 3D-GRE sequence for postcontrast T1-weighted imaging was $3 \mathrm{~mm}$.

\section{Evaluation of MRI}

All MR images were independently reviewed by two radiologists (H.J.C. and Y.K.C., with 10 and 5 years of experience in liver MRI, respectively) on a picture archiving and communication system workstation (Marosis; Marotech, Seoul, Korea). Both radiologists were blinded to the pathologic or clinical details. Nonhypervascular HBPI hypointense nodules were defined as solid nodules with low signal intensity on HBPI, while not showing higher signal intensity than that of the spleen on heavily T2WI. ${ }^{18}$ Only HBPI hypointense nodules with the maximal diameter greater than or equal to $5 \mathrm{~mm}$ were included in this study. This was based on a previous study that none of those nodules with the diameter less than $5 \mathrm{~mm}$ showed malignant transformation during the follow-up. ${ }^{7}$ The HBPI was reviewed first to detect nonhypervascular HBPI hypointense nodules, and then the AP images were reviewed. Hemangiomas or hepatic cysts were excluded from the study based on their enhancement pattern on dynamic phase images and/or hyperintensity on T2WI and heavily T2WI. When discordance in the imaging assessment was present between the two radiologists, final decision was made by consensus.

Table 1. Gadoxetic Acid-Enhanced Liver Magnetic Resonance Imaging Protocol
1. A breath-hold T1-weighted dual-echo (in-phase and opposed-phase) GRE sequence
2. Dynamic 3D fat-saturated T1-weighted GRE sequences, both before and after administration of gadoxetic acid, a liver-specific agent
3. A breath-hold fat-saturated T2-weighted fast spin echo or turbo spin echo sequence
4. A heavily T2-weighted single shot fast spin echo or turbo spin echo sequence
5. Free-breathing DWI using a single shot echo planar imaging sequence

GRE, gradient echo; 3D, three dimensional; DWI, diffusion weighted images. 


\section{Statistical analysis}

Risk factors for the transformation into hypervascular HCCs were analyzed using univariate and multivariate Cox proportional hazard model. Potential risk factors included patient's age and sex, tumor size, hyperintensity on T2WI or DWI, multiplicity of HBPI hypointense nodules, baseline serum level of AFP, initial treatment modality for HCC, history of local therapy of hypervascular HCC nodules, Child-Pugh class, etiology of cirrhosis, and the existence of portal hypertension. The p-values less than 0.05 were regarded as statistically significant.

\section{RESULTS}

\section{Patients and tumors}

During the period from January 2010 to October 2016, 345 consecutive patients with HCC took repetitive MR examinations three or more times over 1 year. Among them, 103 non-hypervascular HBPI hypointense nodules in 60 patients were finally included in the study (Fig. 1). The baseline patient and tumor characteristics are described in Table 2. The diagnosis of HCC was obtained based on the radiological findings in 33 patients, and by the pathological confirmation in the remaining patients.

As an initial treatment, partial hepatectomy and RFA was performed in 27 and 33 patients, respectively. The percentage of patients with portal hypertension were $16.0 \%$ and $33.3 \%$ in patients initially treated by surgical resection or RFA, respectively $(\mathrm{p}=0.127)$. Major and minor resections were performed in 15 and 12 patients, respectively. The number of non-hypervascular hypointense nodules were one in 39 patients, two in nine patients; three in seven patients, four in three patients, and five in two patients. The mean size of a total of 103 non-hypervascular hypointense nodules was $8.5 \pm 3.0 \mathrm{~mm}$ (range, 5.0 to $17.0 \mathrm{~mm}$ ),

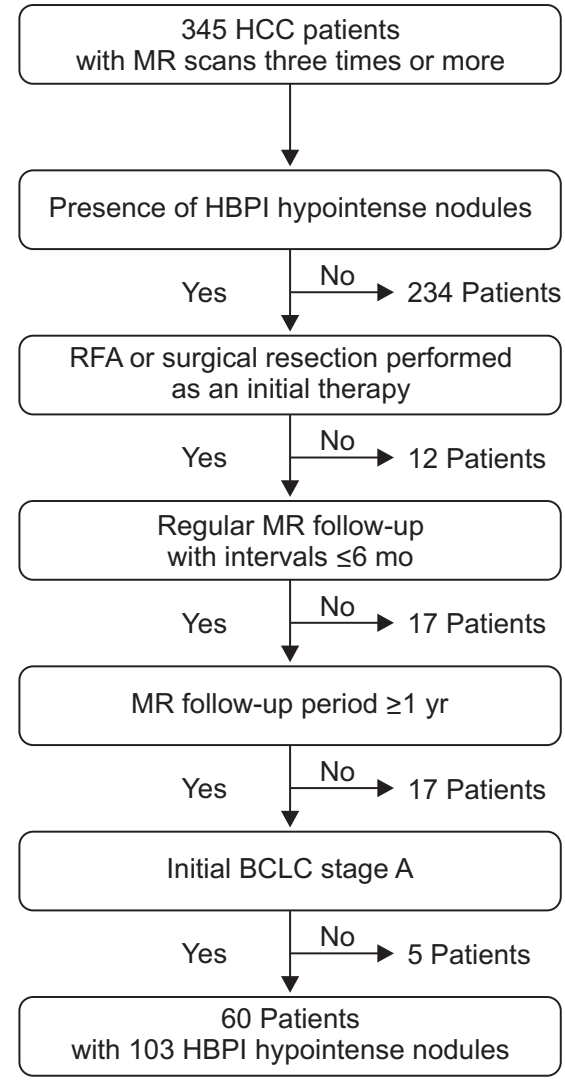

Fig. 1. Diagram illustrating the patients and tumors included in this study.

HCC, hepatocellular carcinoma; MR, magnetic resonance; HBPI, hepatobiliary phase imaging; RFA, radiofrequency ablation; BCLC, Barcelona Clinic Liver Cancer.

Table 2. Baseline Characteristics of the Tumors

\begin{tabular}{lcc}
\hline & Characteristic & Pes/no \\
\hline Age $>$ 65 yr & $60 / 43$ & $58.3 / 41.7$ \\
Male sex & $99 / 4$ & $96.1 / 3.9$ \\
Child-Pugh class B & $2 / 101$ & $1.9 / 98.1$ \\
Portal hypertension & $53 / 50$ & $51.5 / 48.5$ \\
Hepatitis B/C infection/others* & $53 / 28 / 22$ & $51.5 / 28.2 / 20.3$ \\
Tumor diameter $\geq 1$ cm & $25 / 78$ & $24.3 / 75.7$ \\
Multinodular HBPI hypointense nodules & $67 / 36$ & $65.0 / 35.0$ \\
Hyperintensity on T2-weighted images & $11 / 92$ & $10.7 / 89.3$ \\
Hyperintense on DWI (b-value 50) & $14 / 89$ & $13.6 / 86.4$ \\
Hyperintense on DWI (b-value 500-800) & $11 / 92$ & $10.7 / 89.3$ \\
Serum $\alpha$-FP $>$ 10 ng/mL & $38 / 65$ & $36.9 / 63.1$ \\
Previous local therapy for hypervascular HCC in the other site of the liver & $52 / 51$ & $50.5 / 49.5$ \\
Initial partial hepatectomy & $46 / 57$ & $44.7 / 55.3$ \\
\hline
\end{tabular}

The overall numbers of patients and tumors were 60 and 103, respectively.

HBPI, hepatobiliary phase images; DWI, diffusion-weighted images; $\alpha$-FP, $\alpha$ fetoprotein; HCC, hepatocellular carcinoma.

${ }^{*}$ Chronic hepatitis B/chronic hepatitis C/others. 

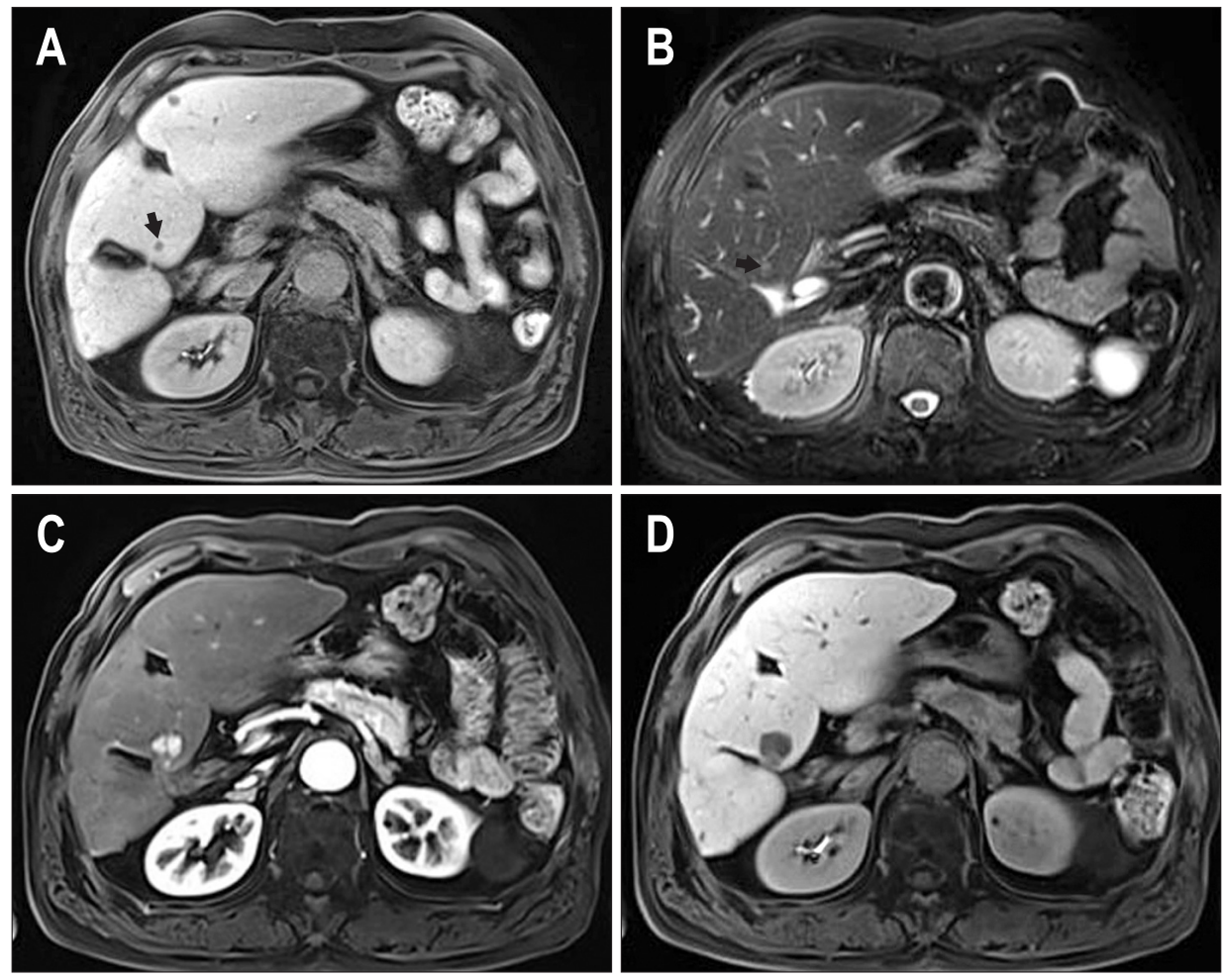

Fig. 2. (A) The axial hepatobiliary phase magnetic resonance (MR) image shows a $0.5-\mathrm{cm}$ well-defined hypointense nodule (arrow). (B) Sixteen months later, the nodule showed hyperintensity in axial T2weighted MR images (arrow). (C) Twenty-five months later, the axial hepatobiliary phase MR (C) and arterial phase images (D) showed that the same nodule had an increased tumor size of $1.7 \mathrm{~cm}$. The nodule was diagnosed as hypervascular hepatocellular carcinoma because of arterial phase hyperintensity and venous washout of contrast enhancement. and the median size was $8.0 \mathrm{~mm}$.

\section{Malignant transformation of HBPI hypointense hepatic nodules}

Overall, 44 hypervascular HCCs (42.7\%) developed in 27 patients among the 103 non-hypervascular hypointense hepatic nodules during follow-up (Fig. 2). The mean and median followup periods were $27.9 \pm 14.2$ months and 23.5 months, respectively. The mean size of 44 non-hypervascular hypointense nodules that progressed into hypervascular HCC were $8.8 \pm 3.2 \mathrm{~mm}$ (range, 5 to $16 \mathrm{~mm}$ ), and the median size was $8.0 \mathrm{~mm}$. For the other 59 non-hypervascular hypointense nodules that did not change into hypervascular HCC, the mean and median size were $8.0 \pm 2.8 \mathrm{~mm}$ (range, 5 to $17 \mathrm{~mm}$ ) and $7.8 \mathrm{~mm}$, respectively. The difference in the diameter between the nodules with or without subsequent malignant transformation was not statistically significant ( $\mathrm{p}=0.705)$. In addition, the mean size of the 44 hypervascular HCCs transformed from pre-existing non-hypervascular hypointense nodules were $14.2 \pm 3.6 \mathrm{~mm}$, and the median size was 13.3 $\mathrm{mm}$. Median doubling time for the 44 tumors was 164.8 days during the last follow-up (range, 34 to 507 days). Among the 23 tumors for which the median doubling times were less than 180 days during the last follow-up, 22 tumors (95.7\%) were finally transformed into hypervascular HCC nodules.

\section{Univariate and multivariate analysis of the prognostic factors for transformation}

Univariate and multivariate analysis revealed that hyperintensity on T2WI or DWI were the two independently adverse predictors of transformation into hypervascular HCCs ( $\mathrm{p}=0.036$ and $\mathrm{p}=0.041$, respectively). Other factors such as tumor size, initial treatment modality (resection or ablation) or the history of prior treatment of hypervascular HCC nodules were not statistically significant (Tables 3 and 4).

All 14 nodules with initial hyperintensity on T2WI and/or DWI were transformed into hypervascular HCCs during follow-up. For these 14 nodules, the 1-, and 2-year transformation rates were $64.3 \%$ and $92.9 \%$, respectively. In contrast, for the 89 nodules without initial hyperintensity on T2WI and/or DWI, the 1-, 2-, and 3-year transformation rates were 9.0\%, 26.0\%, and 39.1\%, respectively. The median times to the transformation into hypervascular HCC were 8.0 months (95\% CI, 3.1 to 12.9 months) and 62.0 months (95\% CI, 31.4 to 92.6 months) for hepatic nodules with or without initial hyperintensity on T2WI and/or DWI, respectively. The difference in the transformation rates between the nodules with or without initial hyperintensity on T2WI and/ or DWI was statistically significant $(\mathrm{p}<0.001)$ (Fig. 3).

\section{Serial changes in the MRI findings during follow-up}

Among the 89 nodules without initial hyperintensity on T2WI or DWI, 12 tumors showed a new finding of hyperintensity on T2WI and/or DWI, and 22 nodules a new finding of hypointensity on PVP or EVP during follow-up, respectively (Fig. 4). All the 12 tumors with a new finding of hyperintensity on T2WI and/or DWI were transformed into hypervascular HCC, and the 1-year transformation rates after the appearance of such signal intensity changes was 91.7\%. For these 12 nodules, median time to malignant transformation was 8.0 months (95\% CI, 6.6 to 9.4 
Table 3. Univariate Analysis of the Prognostic Factors for the Malignant Transformation of Non-Hypervascular MR Hepatobiliary Phase Hypointense Hepatic Nodules

\begin{tabular}{|c|c|c|c|}
\hline & HR (95\% CI) & SE & p-value \\
\hline Age $>65 \mathrm{yr}$ & $2.071(1.081-3.968)$ & 0.332 & $0.028^{*}$ \\
\hline Male sex & $0.407(0.144-1.152)$ & 0.531 & 0.090 \\
\hline Child-Pugh class B & $1.999(0.164-8.737)$ & 1.013 & 0.858 \\
\hline Portal hypertension & $1.109(0.612-2.009)$ & 0.303 & 0.734 \\
\hline Hepatitis C infection & $1.614(0.870-2.995)$ & 0.318 & 0.129 \\
\hline Tumor diameter $\geq 1 \mathrm{~cm}$ & $1.804(0.953-3.416)$ & 0.326 & 0.070 \\
\hline Multiple HBPI hypointense nodules & $0.656(0.356-1.211)$ & 0.312 & 0.178 \\
\hline Hyperintensity on T2WI & $5.752(2.920-11.331)$ & 0.346 & $<0.001^{*}$ \\
\hline Hyperintensity on DWI (b-value 800) & $7.089(3.331-15.087)$ & 0.385 & $<0.001^{*}$ \\
\hline Hypointensity on portal phase images & $0.915(0.437-1.914)$ & 0.377 & 0.813 \\
\hline Intralesional fat deposition & $0.920(0.322-2.632)$ & 0.536 & 0.877 \\
\hline Serum $\alpha-\mathrm{FP}>10 \mathrm{ng} / \mathrm{mL}$ & $1.067(0.585-1.947)$ & 0.307 & 0.833 \\
\hline Previous local therapy for hypervascular HCC & $1.379(0.745-2.551)$ & 0.306 & 0.660 \\
\hline Previous partial hepatectomy & $1.025(0.560-1.875)$ & 0.308 & 0.936 \\
\hline
\end{tabular}

HR, hazard ratio; CI, confidence interval; SE, standard error; HBPI, hepatobiliary phase images; T2WI, T2-weighted images; DWI, diffusionweighted images; $\alpha$-FP, $\alpha$ fetoprotein; HCC, hepatocellular carcinoma.

*Statistically significant.

Table 4. Multivariate Analysis of the Prognostic Factors for the Malignant Transformation of Non-Hypervascular MR Hepatobiliary Phase Hypointense Hepatic Nodules

\begin{tabular}{cccc}
\hline Prognostic factor & HR (95\% CI) & SE & p-value \\
\hline Hyperintensity on T2WI & $3.572(1.083-11.776)$ & 0.609 & $0.036^{*}$ \\
Hyperintensity on DWI & $3.668(1.054-12.768)$ & 0.636 & $0.041^{*}$ \\
Tumor diameter $\geq 1 \mathrm{~cm}$ & $1.858(0.945-3.652)$ & 0.345 & 0.073
\end{tabular}

HR, hazard ratio; CI, confidence interval; SE, standard error; T2WI, T2-weighted images; DWI, diffusion-weighted images.

*Statistically significant.

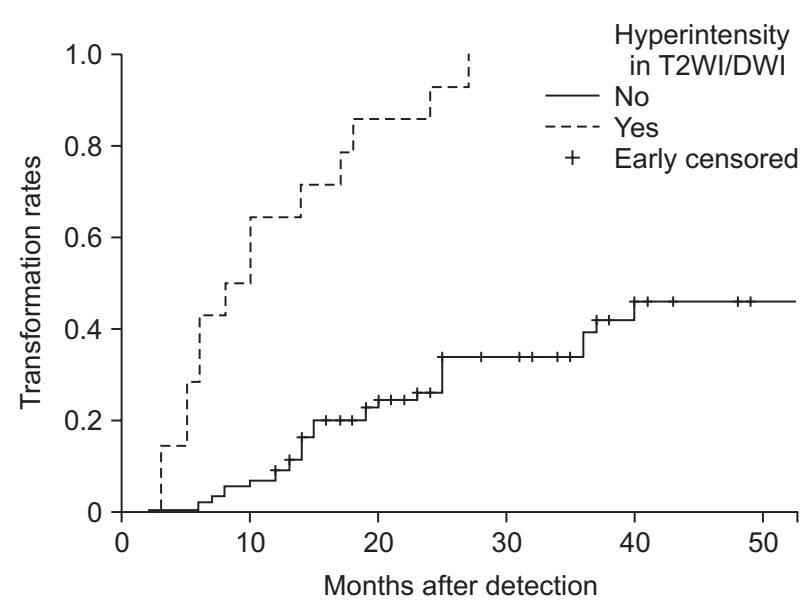

Fig. 3. Comparison of the rates of transformation of non-hypervascular magnetic resonance (MR) hepatobiliary phase hypointense hepatic nodules into hypervascular hepatocellular carcinoma between those with or without hyperintensity according to T2-weighted and/ or diffusion-weighted MR images. The difference between the two subgroups was statistically significant $(\mathrm{p}<0.001)$. DWI, diffusion weighted images. months). Among the 22 nodules with a new finding of hypointensity on PVP or EVP, subsequent findings of hyperintensity on T2WI and/or DWI appeared in five nodules. Among these 22 nodules, 14 nodules (63.6\%) showed malignant transformation, and median time to transformation was 25.0 months (95\% CI, 16.0 to 34.0 months).

Overall, 35 of 44 (79.5\%) hypervascular HCCs transformed from non-hypervascular HB hypointense nodules were associated with baseline or newly appeared hyperintensity on T2WI and/or DWI, or with new signal intensity changes on PVP or EVP images (Fig. 4). In contrast, among the 89 nodules without initial hyperintensity on T2WI and/or DWI, 60 nodules did not show any signal intensity changes described above during follow-up. Only nine of these 60 tumors showed transformation into hypervascular HCCs, and the 1-, 2-, and 3-year transformation rates were $6.7 \%, 14.6 \%$, and $17.8 \%$, respectively.

\section{Overall survival and recurrence after the diagnosis of HCCs}

During follow-up, 11 patients (18.3\%) died. The causes of 


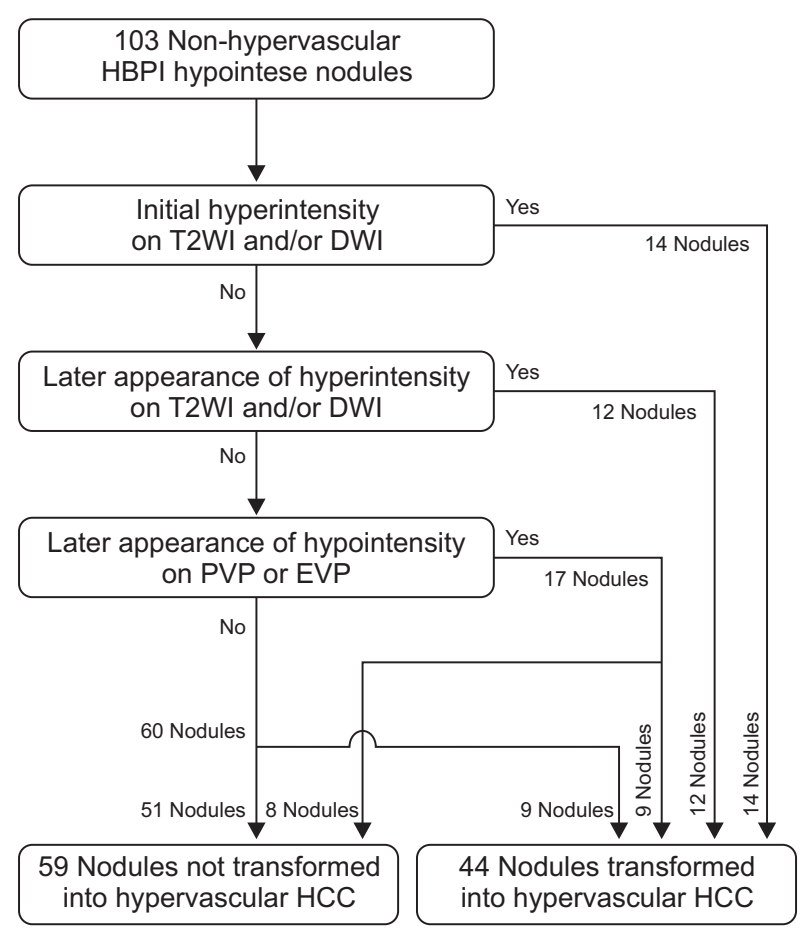

Fig. 4. Diagram illustrating the incidence of the transformation of non-hypervascular magnetic resonance (MR) hepatobiliary phase hypointense hepatic nodules into hypervascular hepatocellular carcinoma according to the initial and serial MR findings.

HBPI, hepatobiliary phase images; T2WI, T2 weighted images; DWI, diffusion weighted images; PVP, portal venous phase; EVP, early venous phase; HCC, hepatocellular carcinoma.

death included liver failure $(n=4)$, progression of HCC $(n=6)$, and pneumonia $(n=1)$, respectively. For the 27 patients initially treated with surgical resection, the estimated 1-, 3-, and 5-year overall survival rates were 100\%, 100\%, and 87.5\%, respectively. For the 33 patients initially treated with RFA, the estimated 1-, 3-, and 5-year overall survival rates were 100\%, 87.2\%, and $79.1 \%$, respectively. The difference in the survival rates was not statistically significant $(\mathrm{p}=0.329)$.

\section{DISCUSSION}

In this study, approximately 80\% (35/44) of malignant transformation of non-hypervascular HBPI hypointense nodules into hypervascular HCC were associated with the initial or later appearance of hyperintensity on T2WI and/or DWI, or new changes in the dynamic MR study suggesting multistep hepatocarcinogenesis. ${ }^{11-13}$ This suggests that most transformation of the borderline malignant hepatic nodules into hypervascular HCC can be predicted based on the initial or serial MR findings.

Previous studies showed that hyperintensity on T2WI or DWI was a potent sign of transformation of HBPI hypointense nodules into overt HCC, as was in this study. ${ }^{711-13,18}$ Large tumor size was known to be a potent sign of transformation of HBPI hypointense nodules into overt HCC, but not in this study. ${ }^{13}$ Other factors known to be significant in the previous studies, such as initial treatment modality, previous local therapy for hypervascular HCC or Child-Pugh class B cirrhosis were not statistically significant in this study. ${ }^{11,18}$ The $42.7 \%$ rate of subsequent hypervascularization was slightly higher than those reported in the previous studies (26.2\% to 31.3\%). ${ }^{7,11}$ This difference may be explained by that cutoff size of small non-hypervascular HBPI hypointense nodule was larger than that in the previous study (5 $\mathrm{mm}$ vs $3 \mathrm{~mm})^{7}$

In this study, most HBPI hypointense nodules did not show initial hyperintensity on T2WI or DWI. It is not definite whether such nodules will be ultimately transformed into hypervascular HCCs. Urgent ablation of such nodules can be an overtreatment, and should not be considered. In contrast, approximately two thirds of HBPI hypointense nodules with initial hyperintensity on T2WI and/or DWI were transformed into hypervascular HCCs within 1 year. We suppose that such nodules should be regarded as malignant rather than premalignant. There may be no reason to delay the treatment for these nodules. Nevertheless, the necessity for the prompt treatment with RFA for the borderline malignant nodules should be decided according to whether additional survival benefits can be obtained by the treatment. If we applied the same simulation model used in our previous study, the expected 5- and 10-year survival benefits were $0.73 \%$ even if the 1-year malignant transformation rate was assumed to be $80 \% .{ }^{19}$ Such a subclinical survival benefit will not justify a prompt treatment even for high risk tumors. ${ }^{20}$

However, some patients with HBPI hypointense nodules may be benefited from the prompt treatment if local ablation treatment is expected to be difficult because of adjacent vascular structures after the nodules are transformed into overt HCC. When liver transplantation is considered, prompt treatment also may be useful to prevent potential microscopic tumor spread after transformation into overt HCC, but further studies will be required. In addition, prompt treatment may be feasible for patients with cancer phobia.

The limitations of the risk classification in this study are as follows. First, ablation of liver tumors less than $1 \mathrm{~cm}$ is technically difficult regardless of the malignant potential. Stratification of malignant transformation potential does not directly lead to the evaluation for the feasibility of prompt ablation therapy. Second, some small HBPI hypointense nodules might have been missed from the database by uncontrolled respiratory movements of patients or misinterpretation of MR images by the radiologists. However, the predictors demonstrated in this study were largely in accord with those of previous studies, including initial hyperintensity on T2WI and/or DWI. ${ }^{7,11,12,18}$ Third, many non-hypervascular HBPI hypointense nodules were less clearly delineated when a 1.5T MR suite was used as compared with 3.0T MR suite. ${ }^{21}$ However, this bias may not be substantial because the cutoff value for the diameter of hepatic nodule was set to be larger than those reported in the literature ( $5 \mathrm{~mm}$ vs 3 
$\mathrm{mm}){ }^{7}$ Future studies based on the results obtained solely from 3.0T MR scanners will be helpful to evaluate the malignant potential of such hepatic nodules. Fourth, larger tumor size may be proved to be a significant predictor in a future large scale study. Finally, the predominant etiology of cirrhosis was chronic viral hepatitis B. In an area where the predominant etiology of cirrhosis was chronic viral hepatitis $\mathrm{C}$, the overall malignant transformation rates may be higher than that in this study.

In summary, most of the malignant transformation of the nonhypervascular HBPI hypointense nodules could be predicted based on the initial or serial follow-up MR findings. However, it is not definite whether HBPI hypointense nodules without initial hyperintensity on T2WI or DWI will be ultimately transformed into hypervascular HCCs. Survival benefits expected from the urgent treatment seems to be subclinical even for the high risk nodules.

\section{CONFLICTS OF INTEREST}

No potential conflict of interest relevant to this article was reported.

\section{ACKNOWLEDGEMENTS}

This study was supported by a VHS Medical Center Research Grant, Republic of Korea (grant number: 16007). We appreciate Mr. Gu Whan Park, the chief MRI technician in VHS Medical Center for his sincere help in this work.

\section{REFERENCES}

1. Takayama T, Makuuchi M, Hirohashi S, et al. Malignant transformation of adenomatous hyperplasia to hepatocellular carcinoma. Lancet 1990;336:1150-1153.

2. Kitao A, Zen Y, Matsui O, Gabata T, Nakanuma Y. Hepatocarcinogenesis: multistep changes of drainage vessels at CT during arterial portography and hepatic arteriography--radiologic-pathologic correlation. Radiology 2009;252:605-614.

3. El-Serag HB, Mason AC. Rising incidence of hepatocellular carcinoma in the United States. N Engl J Med 1999;340:745-750.

4. Choi BI, Lee JM, Kim TK, Dioguardi Burgio M, Vilgrain V. Diagnosing borderline hepatic nodules in hepatocarcinogenesis: imaging performance. AJR Am J Roentgenol 2015;205:10-21.

5. Nakashima 0, Sugihara S, Kage M, Kojiro M. Pathomorphologic characteristics of small hepatocellular carcinoma: a special reference to small hepatocellular carcinoma with indistinct margins. Hepatology 1995;22:101-105.

6. Kim SH, Lim HK, Kim MJ, Choi D, Rhim H, Park CK. Radiofrequency ablation of high-grade dysplastic nodules in chronic liver disease: comparison with well-differentiated hepatocellular carcinoma based on long-term results. Eur Radiol 2008;18:814-821.

7. Lee DH, Lee JM, Lee JY, et al. Non-hypervascular hepatobiliary phase hypointense nodules on gadoxetic acid-enhanced MRI: risk of HCC recurrence after radiofrequency ablation. J Hepatol
2015;62:1122-1130.

8. Inoue T, Kudo M, Komuta M, et al. Assessment of Gd-EOB-DTPAenhanced MRI for HCC and dysplastic nodules and comparison of detection sensitivity versus MDCT. J Gastroenterol 2012;47:10361047.

9. Kobayashi S, Matsui 0, Gabata T, et al. Intranodular signal intensity analysis of hypovascular high-risk borderline lesions of HCC that illustrate multi-step hepatocarcinogenesis within the nodule on Gd-EOB-DTPA-enhanced MRI. Eur J Radiol 2012;81:38393845.

10. Kitao A, Matsui 0, Yoneda N, et al. The uptake transporter OATP8 expression decreases during multistep hepatocarcinogenesis: correlation with gadoxetic acid enhanced MR imaging. Eur Radiol 2011;21:2056-2066.

11. Hyodo T, Murakami T, Imai Y, et al. Hypovascular nodules in patients with chronic liver disease: risk factors for development of hypervascular hepatocellular carcinoma. Radiology 2013;266:480490.

12. Kim YK, Lee WJ, Park MJ, Kim SH, Rhim H, Choi D. Hypovascular hypointense nodules on hepatobiliary phase gadoxetic acidenhanced MR images in patients with cirrhosis: potential of DW imaging in predicting progression to hypervascular HCC. Radiology 2012;265:104-114.

13. Kumada T, Toyoda H, Tada T, et al. Evolution of hypointense hepatocellular nodules observed only in the hepatobiliary phase of gadoxetate disodium-enhanced MRI. AJR Am J Roentgenol 2011;197:58-63.

14. Forner A, Llovet JM, Bruix J. Hepatocellular carcinoma. Lancet 2012;379:1245-1255.

15. Bruix J, Sherman M; Practice Guidelines Committee, American Association for the Study of Liver Diseases. Management of hepatocellular carcinoma. Hepatology 2005;42:1208-1236.

16. An C, Park MS, Kim D, et al. Added value of subtraction imaging in detecting arterial enhancement in small $(<3 \mathrm{~cm})$ hepatic nodules on dynamic contrast-enhanced MRI in patients at high risk of hepatocellular carcinoma. Eur Radiol 2013;23:924-930.

17. Winters SD, Jackson S, Armstrong GA, Birchall IW, Lee KH, Low G. Value of subtraction MRI in assessing treatment response following image-guided loco-regional therapies for hepatocellular carcinoma. Clin Radiol 2012;67:649-655.

18. Toyoda H, Kumada T, Tada T, et al. Non-hypervascular hypointense nodules detected by Gd-EOB-DTPA-enhanced MRI are a risk factor for recurrence of HCC after hepatectomy. J Hepatol 2013;58:1174-1180.

19. Cho YK, Wook Chung J, Kim Y, Je Cho H, Hyun Yang S. Radiofrequency ablation of high-grade dysplastic nodules. Hepatology 2011;54:2005-2011.

20. Midorikawa Y, Takayama T, Shimada K, et al. Marginal survival benefit in the treatment of early hepatocellular carcinoma. J Hepatol 2013;58:306-311.

21. Chang KJ, Kamel IR, Macura KJ, Bluemke DA. 3.0-T MR imaging of the abdomen: comparison with $1.5 \mathrm{~T}$. Radiographics 2008;28:1983-1998. 